\title{
Contaminación del agua por plaguicidas en un área de Antioquia
}

\section{Water pollution caused by pesticides in an area of Antioquia}

\author{
Flor Á. Tobón-Marulanda ${ }^{1}$, Luís A. López-Giraldo² y Ramón E.Paniagua-Suárez ${ }^{3}$
} 1 Facultad de Química Farmacéutica, Grupo de investigación Unipluriuniversidad, Universidad de
Antioquia. Medellín, Colombia. jvm@une.net.co.
2 Facultad de Enfermería, Universidad de Antioquia. Medellín, Colombia. rush@une.net.co.
3 Facultad Nacional de Salud Pública, Universidad de Antioquia. Medellín, Colombia.
epania@guajiros.udea.edu.co

Recibido 9 Junio 2009/Enviado para Modificación 14 Marzo 2010/Aceptado 18 Abril 2010

\section{RESUMEN}

Objetivo Determinar la frecuencia de uso de plaguicidas en la producción agropecuaria, porcícola y avícola, para comprender su influencia en el recurso hídrico en la vereda Monterredondo del municipio San Pedro de los Milagros (Antioquia) y establecer un diagnostico de su calidad.

Métodos Se aplicó una encuesta domiciliaria al $100 \%$ de las familias de la vereda, sobre el uso de productos químicos y se realizaron dos análisis, el fisicoquímico y el microbiológico a tres muestras de agua en el laboratorio de Ingeniería Ambiental de la Universidad de Antioquia bajo métodos estandarizados.

Resultados La quebrada El Hato presenta una contaminación por Coliformes de 1 $600 \times 103 \mathrm{NMP} / 100 \mathrm{ml}$ y por E.coli de 220x10 NMP/100ml. La quebrada Fray Juana presenta un grado de contaminación de $1600 \times 103$ NMP/100ml de Coliformes. Se encontró igualmente una contaminación de las fuentes hídricas por el uso de diversos plaguicidas como: Lorsban $\AA$ (Clorpirifos), Ráfaga $\AA$ (Clorpirifos), Látigo $\AA$ (Clorpirifos), Neguvón® (Metrifonato), Furadán® (Carbofurán). Con insecticidas como, Ganabaño® (Cibermetrina) y desinfectantes como, Límpido® (Hipoclorito de sodio $6 \%$ ).

Conclusión La producción agropecuaria, porcícola y avícola en la vereda, está generando impactos negativos en el recurso hídrico, por la disminución y la contaminación de éste. Las aguas de las muestras analizadas, no son aptas para el consumo de seres vivos.

Palabras Clave: Agua, ambiente, contaminación (fuente: DeCS, BIREME).

\section{ABSTRACT}

Objective Determining the frequency of pesticide use in agricultural, pig and poultry production to understand its influence on water resources in the village of Monterredondo in the municipality of San Pedro de Los Milagros (Antioquia) and diagnosing its quality. 
Methods $100 \%$ of the households in the village were surveyed regarding chemical use. Physicochemical and microbiological analysis of three water samples was carried out in the Universidad de Antioquia's Environmental Engineering laboratory using standardised methods.

Results The Hato stream was contaminated by $1,600 \times 103 \mathrm{NMP} / 100 \mathrm{ml}$ coliforms and $220 \times 10 \mathrm{NMP} / 100 \mathrm{ml}$ E. coli. The Fray Juana stream had $1,600 \times 103 \mathrm{NMP} / 100 \mathrm{ml}$ coliform contamination. Water sources were also contaminated by the use of pesticides such as Lorsban (chlorpyriphs), Burst (chlorpyriphs), Whip (chlorpyriphs), Neguvon (metriphonate) and Furadan (carbophuran) and insecticides like Ganabaño (cypermethryn) and disinfectants and Limpido (6\% sodium hypochlorite).

Conclusion Agricultural, pig and poultry production in the village has been producing a negative impact on water resources due to its reduced availability and pollution. The water from the samples analysed here was not suitable for human consumption.

Key Words: Water, environment, pollution (source: MeSH, NLM).

$\mathrm{E}$ 1 agua es el componente fundamental en mayor cantidad de los organismos de los seres vivos, indispensable para la mayoría de las funciones vitales. Es un elemento esencial en la producción agropecuaria, porcícola y avícola; por lo cual, su calidad incide directamente en la calidad de los productos alimenticios y debe cumplir con parámetros de calidad para ser consumida por seres vivos (1). El agua requiere estar libre de elementos tóxicos, que pueden acumularse en los tejidos y fluidos de los animales y plantas de granja, convirtiéndose en productos alimenticios nocivos.

En la vereda Monterredondo existen dos microcuencas: 1. La quebrada Fray Juana, que divide los terrenos de la Hacienda La Montaña y sus aguas se utilizan como fuente de abastecimiento para sus unidades de producción. Estas aguas, son tratadas en una planta de potabilización ubicada en su predio. 2. La quebrada El Hato, que recibe las aguas de la quebrada Fray Juana, dentro de la hacienda. Ambas microcuencas son contaminadas por tres factores fundamentales: a) descarga directa de aguas residuales, resultado del lavado de la infraestructura productiva; b) descarga directa de residuos sólidos y c) utilización inadecuada de plaguicidas y pesticidas tóxicos, contaminantes de dichas fuentes hídricas que merecen particular atención, como son los compuestos órganoclorados, organofosforadas y los carbamatos (1-7).

En la calidad biosfera, donde tienen lugar la totalidad de las actividades antrópicas, intervienen factores fisicoquímicos y microbiológicos del agua. Así, el estudio de la calidad de los procesos productivos es importante para la comprensión de los efectos tóxicos de elementos presentes en los efluentes 
que inciden en la situación de salud pública de las comunidades, que se sirven de aguas contaminadas $(8,9)$.

En la Vereda Monterredondo, es común la exposición crónica a plaguicidas, cuyos efectos en la salud surgen a largo plazo. De las enfermedades dermatológicas, respiratorias y del sistema digestivo, manifestadas por los pobladores de la vereda, no se tiene la certeza del compuesto químico específico causante, ni de su concentración, ni del tiempo de exposición. Según la literatura científica, los pesticidas y los plaguicidas, utilizados a largo plazo, generan efectos tóxicos como: malformaciones, carcinomas, parálisis, daños nerviosos, mutaciones, infertilidad, teratogénesis y convulsiones, entre otros (9-12).

El objetivo de esta investigación fue determinar la frecuencia de uso de plaguicidas y valorar la calidad del agua de fuentes hídricas de la vereda Monterredondo.

\section{MATERIALES Y MÉTODOS}

Se realizó un estudio de tipo descriptivo con enfoque cuantitativo mediante una encuesta domiciliaria aplicada a 73 hogares de la vereda (100\%) sobre la frecuencia de uso de plaguicidas. Para el análisis de los datos, se emplearon tablas de frecuencia elaboradas con el programa de estadístico, Statistical Package for the Social Sciences (SPSS), versión 15.0, para obtener las distribuciones porcentuales. Se realizaron pruebas fisicoquímicas y microbiológicas a dos muestras de agua de las quebradas Fray Juana y El Hato y a una muestra de agua de la planta de tratamiento de la Hacienda La Montaña de la Universidad de Antioquia; efectuadas por el Laboratorio de Análisis de Aguas de Ingeniería Sanitaria y Ambiental de la Facultad de Ingeniería de la Universidad de Antioquia, mediante técnicas estandarizadas para su recolección y análisis (13-15).

\section{RESULTADOS}

La Tabla 1 muestra la distribución de frecuencia del uso de productos químicos más utilizados por la población de la vereda Monterredondo. Preocupa el uso de manera frecuente de plaguicidas de toxicidad alta como: Lorsban ${ }^{\circledR}$ (Clorpirifos), Ráfaga ${ }^{\circledR}$ (Clorpirifos), Látigo ${ }^{\circledR}$ (Clorpirifos), Neguvon ${ }^{\circledR}$ (Metrifonato), Furadán ${ }^{\circledR}$ (Carbofurán), Ganabaño® (Cipermetrina) y el Límpido® (Hipoclorito de sódio $6 \%$ ), desinfetante. 
Los datos de la Tabla 2 permiten observar que si bien las propiedades fisicoquímicas de las tres muestras de agua analizadas, se encuentran por debajo del valor máximo permitido por la Resolución 2115 de 2007, el valor de la Demanda Química de Oxigeno para la muestra M3, agua tratada, debiera ser cero. Todos los valores de las propiedades fisicoquímicas de las tres muestras analizadas, son semejantes entre sí.

Tabla 1. Distribución porcentual de los productos químicos de uso más frecuente por las familias encuestadas en la vereda Montenegro del municipio de San Pedro de los Milagros, 2008

\begin{tabular}{|c|c|c|c|}
\hline Producto & Toxicidad & Uso & $\begin{array}{c}\text { Frecuencia }^{*} \\
\%\end{array}$ \\
\hline Límpidoß (Hipoclorito de sódio 6\%) & III Media & Desinfectante & 82 \\
\hline Lorsban $®$ (Clorpirifos) & I Alta & Plaguicida & 47 \\
\hline Urea® ( Nitrógeno) & IV Baja & Fertilizante de plantas & 34 \\
\hline Ráfaga ${ }^{\circledR}$ (Clorpirifos) & I Alta & Plaguicida & 33 \\
\hline Látigoß (Clorpirifos) & I Alta & Plaguicida & 30 \\
\hline 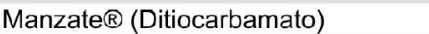 & Mediana & Plaguicida & 27 \\
\hline Curagan $®(F e n o l)$ & Alta acuática & Antiinfeccioso & 26 \\
\hline Calmafox® (medicamento) & No aplica & Aporte de vitaminas & 25 \\
\hline Oxitetraciclina (medicamento) & Baja & Antiinfeccioso & 22 \\
\hline Triple quince $®(N, P, K)$ & No aplica & Fertilizante de plantas & 22 \\
\hline Vitaminas Complejo B & No aplica & Aporte de vitaminas & 19 \\
\hline Lutalise $\otimes\left(P G F_{2}\right.$ alfa) & No aplica & Estimular ovulación & 18 \\
\hline Neguvon® (Metrifonato) & I Alta & Plaguicida & 16 \\
\hline Ganabaño® (Cipermetrina) & Extrema Alta & Insecticida & 15 \\
\hline Triatox $\otimes($ Amitraz) & Media & Ectoparasitosis & 14 \\
\hline Panacur $(\mathbb{B})$ (Fenbendazol ) & Baja & Nemanticida & 12 \\
\hline Karate $($ (D-Cyalotrina) & Вaja & Plaguicida & 11 \\
\hline Barricada@ (Cipermetrina) & Baja & Plaguicida & 11 \\
\hline Engeo $囚$ (Neonicotinoide+piretroide) & Вaja & Plaguicida & 10 \\
\hline Furadán® (Carbofurán) & Extrema Alta & Plaguicida & 8 \\
\hline Tetraciclina & No aplica & Antibiótico & 8 \\
\hline Roundup® (Glifosato) & Baja & Herbicida Total & 8 \\
\hline Vitamina Mk & No aplica & Aporte de vitaminas & 8 \\
\hline Lepecid® (Clorpirifos) & III Mediana & Larvicida y antiséptico & 7 \\
\hline Piretroides & Baja & Insecticida & 7 \\
\hline Ivermectina Genfar® & Extrema Alta & Ectoparasitosis & 6 \\
\hline Pirestar $(B)$ (Permetrina) & III & Plaguicida & 6 \\
\hline Oxitocina & No aplica & Hormonas & 6 \\
\hline Glifosato & Baja & Herbicida total & 4 \\
\hline
\end{tabular}

La Tabla 3 muestra que los resultados del análisis microbiológico están por encima de los valores máximos permitidos (Resolución 2115 de 2007), indicando que la calidad del agua no cumple con la norma. La quebrada El Hato $\left(\mathrm{M}_{1}\right)$ presenta el grado más alto de contaminación por Coliformes y E.coli, seguida por las muestras de agua de la quebrada Fray Juana $\left(\mathrm{M}_{2}\right)$ y de la muestra proveniente de la planta de tratamiento de la Hacienda la Montaña $\left(\mathrm{M}_{3}\right)$. 
Tabla 2. Pruebas fisicoquímicas de tres muestras de agua de la vereda Monterredondo de San Pedro de los Milagros-Antioquia, 2008

\begin{tabular}{|c|c|c|c|c|c|}
\hline Parámetro & Unidad & $M_{1}$ & $\mathrm{M}_{2}$ & $M_{3}$ & Valor máximo \\
\hline DQO & $0.2 \mathrm{mg} / \mathrm{l}$ & 33.40 & 20.04 & 66.78 & 350 \\
\hline DBO & $02 \mathrm{mg} / /$ & 15.70 & 8.55 & 57.0 & 60 \\
\hline OD & $\mathrm{O} 2 \mathrm{mg} / \mathrm{I}$ & 5.60 & 6.20 & 5.70 & NR \\
\hline Sólidos Totales & $\mathrm{mg} / \mathrm{l}$ & 130.00 & 108.00 & 100 & 500 \\
\hline Sólidos Suspendidos & $\mathrm{mg} / \mathrm{l}$ & 46.00 & 68.00 & 36 & NR \\
\hline Sólidos Disueltos & $\mathrm{mg} / \mathrm{l}$ & 84.00 & 40.00 & 64 & NR \\
\hline Alcalinidad & $\mathrm{mg}$ de $\mathrm{CaCO}_{3} / \mathrm{l}$ & 14.80 & 12.00 & 8.4 & 200 \\
\hline Dureza Total & $\mathrm{mg}$ de $\mathrm{CaCO}_{3} / \mathrm{l}$ & 26.40 & 29.20 & 18.4 & 300 \\
\hline $\mathrm{pH}$ & Unidad de $\mathrm{pH}$ & 6.70 & 6.72 & 6.76 & $6.5-9.0$ \\
\hline Cloruros & $\mathrm{mg} \mathrm{Cl}-1 \mathrm{l}$ & 2.40 & 3.10 & 4.40 & 250 \\
\hline Color Verdadero & UPC & 28.00 & 30.00 & 28.0 & 15 \\
\hline Conductividad & microsiemens/cm & 53.50 & 54.30 & 54.1 & $50-1000$ \\
\hline Nitrógeno Total & $\mathrm{mg} \mathrm{NH} / 3-\mathrm{N} / \mathrm{l}$ & 3.86 & 2.80 & 2.80 & 40 \\
\hline Fósforo Total & $\mathrm{mg} \mathrm{P} / \mathrm{l}$ & 0.100 & $<0.00439$ & 0.078 & 10 \\
\hline
\end{tabular}

Tabla 3. Pruebas microbiológicas de tres muestras de agua de la vereda Monterredondo de San Pedro de los Milagros-Antioquia, 8

\begin{tabular}{|c|c|c|c|c|c|}
\hline $\begin{array}{l}\text { Indicador de } \\
\text { Contaminaçón }\end{array}$ & Unidades & $M_{1}$ & $M_{2}$ & $M_{3}$ & Valor máximo \\
\hline Coliformes $^{*}$ & $\begin{array}{l}\text { NMP por } \\
100 \mathrm{ml}\end{array}$ & $1.600 \times 10^{3}$ & $>1.600 \times 10^{3}$ & NR & $\begin{array}{c}<\text { de } 1 \text { m.o. en } \\
100 \mathrm{~cm} 3\end{array}$ \\
\hline E. coli-Múltiples & $\begin{array}{c}\text { NMP por } \\
100 \mathrm{ml}\end{array}$ & $220 \times 10$ & NA & NR & $\begin{array}{c}<\text { de } 1 \text { m.o. en } \\
100 \mathrm{~cm}^{3}\end{array}$ \\
\hline
\end{tabular}

\section{DISCUSIÓN}

La producción agropecuaria, porcícola y avícola en zona de estudio, está generando impactos negativos en el recurso hídrico. Igualmente, está afectando el medio ambiente al generar compactación y contaminación del suelo, erosión, contaminación del aire, producción de residuos sólidos y proliferación de vectores. En la planta de tratamiento de la Hacienda la Montaña se lleva un registro muy superficial sobre la calidad de agua, que básicamente mide $\mathrm{pH}$ y Cloro. Además, no se cuenta con un método, después de su tratamiento, para comprobar la veracidad de los indicadores de la evaluación. El tanque de almacenamiento de agua de la Hacienda la Montaña presenta condiciones insalubres, dado que en él se observa presencia de lama y moho.

Los resultados de los análisis fisicoquímicos y microbiológicos indican que el tratamiento al agua en la Hacienda la Montaña $\left(\mathbf{M}_{3}\right)$, no es efectivo. Es necesario verificar los indicadores de su calidad, a la luz de la normatividad 
vigente. El análisis microbiológico de las tres muestras, revela que estas aguas no son aptas para el consumo de seres vivos y requieren de vigilancia especial para su uso. El excesivo uso de plaguicidas en la Vereda Monterredondo, puede estar impactando de manera negativa, la salud pública de la población expuesta. En la literatura se reportan estudios de genotoxicidad, intoxicaciones y muertes producidas por el mal uso de plaguicidas. Muchos de estos compuestos son sustancias lipofílicas que pueden migrar a través de la cadena alimenticia y almacenarse en el tejido adiposo y en el Sistema Nervioso Central. Por esto, se encuentran niveles elevados de estos químicos en el tejido adiposo de pacientes con cáncer (16-20).

Algunos productos químicos tóxicos utilizados por esta población son: Límpido ${ }^{(H i p o c l o r i t o ~ d e ~ s o d i o ~} 6 \%$ ); Úrea (compuesto ureico de nitrógeno); Roundup ${ }^{\circledR}$ (Glifosato), herbicida total no selectivo de amplio espectro para eliminar hierbas y arbustos perennes. Según estudios reportados por Yoke $\mathrm{CH}$ (2005), la exposición al Glifosato es un factor de riesgo que puede afectar el ácido desoxirribonucleico-ADN, a cientos de células. En contraste, estudios de la Organización Mundial de la Salud (2006), lo cataloga de baja toxicidad. Concierne a las autoridades públicas valorar el riesgo/beneficio y tener en cuenta precauciones para su uso racional (21).

En el tratamiento del agua de la Hacienda La Montaña, el objetivo de la dosis de coagulante del agua (Sulfato de Aluminio), es precipitar todos los sólidos, haciendo que el color disminuya. Pero, el valor del color de las tres muestras analizadas es muy similar; así como los valores de sólidos totales, sólidos suspendidos, sólidos disueltos y de la alcalinidad, entre otros parámetros fisicoquímicos (13-14, 22-23).

El análisis microbiológico es un indicador de contaminación fecal en el agua de consumo de seres vivos, con un alto riesgo de contaminación microbiana por bacterias Gram Negativas en forma bacilar que fermentan la lactosa (Coliformes) y de la bacteria Escherichia coli, bacilo aerobio, Gram Negativo y no esporulada. Su presencia, en la mayoría de los casos, revela contaminación concomitante con otros gérmenes termorresistentes a temperaturas superiores a $100^{\circ} \mathrm{C}$, como huevos de helmintos. Por tal razón, no es confiable la técnica de hervir estas aguas para garantizar su potabilidad, como lo hacen algunas personas en la vereda Monterredondo. Teniendo en cuenta que los efectos de la contaminación del agua por gérmenes en los seres vivos, son de diversas características e impacto sobre la salud pública. Por tal razón, el control y la evaluación de enterobacteriáceas en el agua potable y en el agua cruda de uso 
humano, debe ser un objetivo primordial en investigaciones sobre la calidad de las mismas (24-25).

La quebrada El Hato $\left(\mathrm{M}_{1}\right)$ presenta el grado más alto de contaminación por Coliformes y E.coli, seguida por las muestras de agua de la quebrada Fray Juana $\left(\mathrm{M}_{2}\right)$ y de la muestra de la planta de tratamiento de la Hacienda la Montaña $\left(\mathrm{M}_{3}\right)$. Entre las posibles causas de esta contaminación están: la materia fecal procedente de bovinos, porcinos y galpones que por efecto de la escorrentía se transporta a estas fuentes hídricas y las aguas residuales de las viviendas. No se cuenta con una infraestructura adecuada para el transporte de las aguas residuales, ni instalaciones de tratamiento que cumplan con la normatividad, lo cual se constituye en un riesgo ambiental para la salud de la comunidad.

Una fortaleza del estudio es el cruce de la información proporcionada por los campesinos sobre el uso de plaguicidas con el análisis fisicoquímico y microbiológico del agua. La debilidad del estudio, es que no se cuenta con información longitudinal de muestras del agua tomadas a través de un periodo determinado de tiempo.

Teniendo en cuenta los resultados expuestos, se debe implementar en la Hacienda la Montaña un Plan de Manejo Ambiental, con especial énfasis en el manejo adecuado de los efluentes y la conservación de las fuentes de agua, de conformidad con la legislación vigente en materia ambiental; además de modernizar su planta de tratamiento •

Agradecimientos: Los autores agradecen a la Vicerrectoría de Extensión de la Universidad de Antioquia, que financió esta investigación. Al Laboratorio de Análisis de Aguas de Ingeniería Sanitaria y Ambiental de la Facultad de Ingeniería de la Universidad de Antioquia, por su apoyo técnico-científico. A las estudiantes Mary Luz Flórez Castaño y Lissette Marcela Jaramillo Díaz de Facultad de Química Farmacéutica y Yuly Viviana Carvajal Sánchez de la Facultad de Enfermería.

\section{REFERENCIAS}

1. Aleem A, Malik A. Genotoxicity of water extracts from the River Yamuna at Mathura. Environmental toxicology. 2003; 18 (2):69-77.

2. Cuenca $P$, Ramírez V. Aberraciones cromosómicas en trabajadoras expuestas a plaguicidas. Revista Biología Tropical. 2004; 52 (3): 623-628.

3. Meléndez I, Zuleta M, Marín I, Calle J, Salazar D. Actividad mutagénica de aguas de consumo humano antes y después de clorar en la planta de Villa Hermosa, Medellín. latreia. 2001; 14 (3): 167-174.

4. Stahl RG. The genetic toxicology of organic compounds in natural waters and wastewaters, Ecotoxicology Environmental Safety. 1991; 22 (1):94-125. 
5. Taiganides EP. 1992. [Internet]. Pig Waste Management and Recycling. The Singapore Experience. International Development Research. Ottawa. Disponible en: http:// www.nzdl.org/fast-cgi-bin/library?e=d-00000-00---off-0envl--00-0----0-10-0---0---0direct-10---4-----0-1|--11-en-50---20-about---00-0-1-00-0-0-11-1-0utfZz-8$00 \& \mathrm{a}=\mathrm{d} \& \mathrm{c}=\mathrm{env} \& \mathrm{cl}=\mathrm{CL} 1.5 \& \mathrm{~d}=\mathrm{HASH} 59 \mathrm{ae} 0385 \mathrm{a9209} \mathrm{c} 06 \mathrm{baa} 73 \mathrm{a} .4$ Consultado: Febr 14, 2009.

6. Córdoba D. Toxicología. 5 ed. Bogotá D.C.: El Manual Moderno; 2006. p. 858.

7. Rivero O, Rizo P, Ponciano G, Olaiz G. Daños a la salud por plaguicidas. México: El manual moderno; 2001. p. 488.

8. Castro R, Ramírez V, Cuenca P. Análisis de micronúcleos y otras anormalidades nucleares en el epitelio oral de mujeres expuestas ocupacionalmente a plaguicidas. Revista Biología Tropical. 2004; 52: 207-217.

9. García M, Toalá D [Internet]. 2003. Evaluación de la contaminación de residuos de plaguicidas órganoclorados en el cultivo de Melón (Cucumis melo L.) en localidades de la Comarca Lagunera. Tesis para optar al título de agrónomo general de la uaaan-ul, México: 1-5. Disponible en: http:/www.uaaan.mx/Dirlnv/Rdos2003/cuencas/cucumis.pdf Consultado: 25 junio 2008.

10. MICROMEDEX (Drug evaluation Monographs). Centro de Información de medicamentos, drogas y alimentos-CIDUA. Universidad de Antioquia. Facultad de Química Farmacéutica, Medellín, Colombia; 1998.

11. Velasco A, Del Barrio L, Serrano J, Martínez R, Cadavid M. Farmacología Fundamental. Primera edición. Madrid: McGraw-Hill Interamericana; 2003. pp. 1019.

12. Rang H, Dale M, Ritter J, Moore P. Farmacología. 5 ed. Madrid: Elsevier; 2004. p. 885.

13. República de Colombia. Ministerio de Salud y de la Protección Social, Ministerio de Ambiente, Vivienda y Desarrollo Territorial 2007. Decreto 1575, Sistema de Protección y Control de la Calidad del Agua para Consumo Humano. Bogotá D.C.: Ministerio de Ambiente, Vivienda y Desarrollo Territorial; mayo 09 de 2007.

14. República de Colombia. Ministerio de Salud y de Protección Social. Bogotá D.C.: Resolución 2115. Características, instrumentos básicos y frecuencias del sistema de control y vigilancia para la calidad del agua para consumo humano; 2007.

15. Eaton A, Clesceri L, Rise E, Greenberg A. Standard methods for the examination of water and wastewater. 21 ed. Washington: American Public Health; 2005. p. 1368.

16. Toro G, Cárdenas O, Varona M. 2002. Neurotoxicidad (III parte) plaguicidas. Acta Neurológica Colombiana. 2002; 18: 32-50.

17. Vindas R, Ortiz F. Genotoxicidad de tres plaguicidas utilizados en la actividad bananera en Costa Rica. Revista Biología Tropical. 2004; 52 (3): 601-609.

18. Zuleta M, Uribe Y, Valencia C, Vargas H, Orozco LY, López C. 2004. Contribución de la contaminación y cloración en la mutagenicidad, genotoxicidad y presencia de mutágenos en agua potable. Actualidades Biológicas. 2004;26 (81): 125-136.

19. Toro G, Cárdenas O, Varona M. Neurotoxicidad (III parte) plaguicidas. Acta Neurológica Colombiana. 2002; 18: 32-50.

20. Uribe M. Neurotoxicología. Bogotá D.C.: Asociación Colombiana Neurológica; 2001. pp. 117-141.

21. Yoke $\mathrm{CH}$ 2005. Inquietantes efectos del Roundup $\AA$ (Glifosato) en los seres humanos y el ambiente. [Internet].p.7. Disponible en: http://www.redtercermundo.og.uy/ texto_completo.php?id=2717. Consultado: 25 junio 2008.

22. Rodríguez J, Galvín R. Fisicoquímica de aguas. Madrid: Díaz De Santos S.A.; 1999. pp. 19-14.

23. Centro Panamericano de Ingeniería y Ciencias del Ambiente 1981. Teoría, Diseño y Control de los Procesos de Clarificación del Agua. 13 ed. Lima (Perú): Oficina Sanitaria Panamericana de la Salud; 1981. pp. 18-12.

24. Cincinnati. United States Environmental Protection Agency. Methods for Chemical Analysis of Water and Wastes; 1983.

25. Organización Mundial de la Salud 2006. Guías para la Calidad del Agua Potable. Aspectos Químicos [Internet]. 3 ed. Suiza: OMS. p. 398. Disponible en: http://www.who.int/ water_sanitation_health/dwq/guidelines/es/ Consultado: Dic 28, 2008. 